\title{
Futuximab/Modotuximab Mixture
}

National Cancer Institute

\section{Source}

National Cancer Institute. Futuximab/Modotuximab Mixture. NCI Thesaurus. Code C91381.

A mixture of two recombinant IgG1 antibodies directed ag ainst different epitopes in the epidermal growth factor receptor (EGFR) extracellular domain III, with potential antineoplastic activity. Anti-EGFR monoclonal antibody mixture Sym004 binds to the extracellular domain of EGFR, thereby preventing ligand binding. This may prevent activation and subsequent dimerization of the receptor; the decrease in receptor activation may result in an inhibition of downstream ERK and JNK signaling pathways and thus inhibition of EGFR-dependent tumor cell proliferation and metastasis. In addition, binding of Sym004 to EGFRs causes EGFR internalization and degradation. EGFR, a receptor tyrosine kinase, often is overexpressed on the cell surfaces of various solid tumor cell types. 